\title{
Comparative Evaluation of Collagen Fibers in Odontogenic Keratocyst and Unicystic Ameloblastoma
}

\author{
${ }^{1}$ Vaidhehi N Nayak, ${ }^{2}$ Mandana Donoghue, ${ }^{3}$ Praveen S Basandi, ${ }^{4} \mathrm{M}$ Selvamani \\ ${ }^{1}$ Senior Lecturer, Department of Oral and Maxillofacial Pathology, KLE Dental College, Bengaluru, Karnataka, India \\ ${ }^{2}$ Professor and Head, Department of Oral and Maxillofacial Pathology and Microbiology \\ College of Dental Sciences, Davangere, Karnataka, India \\ ${ }^{3}$ Assistant Professor, Department of Oral and Maxillofacial Pathology and Microbiology \\ College of Dental Sciences, Davangere, Karnataka, India \\ ${ }^{4}$ Senior Lecturer, Department of Oral Pathology and Microbiology, College of Dental Sciences, Davangere, Karnataka, India
}

Correspondence: Vaidhehi N Nayak, Senior Lecturer, Department of Oral and Maxillofacial Pathology, KLE Dental College Bengaluru Karnataka, India, e-mail: vaidhehinayak2011@ gmail.com

\section{ABSTRACT}

Background: Odontogenic keratocyst (OKC) is an aggressive cyst with neoplastic behavior and unicystic ameloblastoma (UA) is a neoplasm with cyst like behavior. Both odontogenic keratocyst and unicystic ameloblastoma show biologic behavior unlike other lesions in their respective groups. In the present study, the biological behavior of these lesions were assessed by studying the collagen fibers in their walls using picrosirius red (PSR) polarization method.

Methods: Collagen fibers in 20 cases of OKC and 20 cases of UA were studied histologically by staining sections with picrosirius red dye and examining them by polarizing microscopy. Polarization colors of the collagen fibers of the lesions were recorded according to their width.

Results: While no differences were seen between the polarization colors of thin fibers $(<0.8 \mu)$ in both odontogenic keratocyst and unicystic ameloblastoma, the polarization colors of thick fibers of odontogenic keratocyst were significantly more greenish-yellow as compared to the unicystic ameloblastoma which were predominantly yellowish-red.

Conclusion: These findings suggest that odontogenic keratocyst is a more aggressive lesion than unicystic ameloblastoma by means of identifying abnormally packed collagen fibers in odontogenic keratocyst but not in unicystic ameloblastoma. Thus, the nature of collagen fibers as studied by the picrosirius red polarization method may be useful as a diagnostic tool to differentiate between the two lesions.

Keywords: Odontogenic keratocyst, Unicystic ameloblastoma, Collagen fibers, Picrosirius dye, Polarization.

\section{INTRODUCTION}

The benign cystic neoplasm is an entity in which the cystic lesion exhibits a neoplastic behavior clinically. Odontogenic keratocyst $(\mathrm{OKC})$ is proved to bel ong to this category. ${ }^{1} \mathrm{On}$ the contrary, unicystic ameloblastoma (UA) is a neoplasm with cyst like features clinically. ${ }^{2}$ These lesions with their contradictory behavior pose difficulties in establishing standard treatment protocols.

The epithelia of these lesions have been investigated extensively in regards to their role in the proliferative and aggressive behavior of the lesions. However, the role of the connective tissue wall in their behavior has not been studied as extensively.

Collagen is an essential part of connective tissue as a whole and fibrous wall of cystic lesions especially. It is demonstrated by picrosirius red dye staining combined with polarization microscopy. This method permits the evaluation of the nature of the collagen fibers in addition to their thickness. ${ }^{3}$

The present study is aimed at examination and comparison of collagen fibers in the connective tissue of OKC and UA with an objective of identifying any prognostic role played by connective tissue in the behavior of these lesions.

\section{METHODS}

A total of 20 cases of OKC and UA were retrieved from the archives of the Department of O ral Pathology and M icrobiology, College of D ental Sciences, B apuji Dental College and Hospital, Davangere and Government Dental College, Chennai. Two paraffin embedded sections ( $6 \mu \mathrm{m}$ thick) were obtained for each case. The diagnosis of each case was confirmed by examining one section stained by hematoxylin and eosin using conventional light microscope. The second section was then stained using PSR.

The PSR stained sections showing collagen fibers within the connective tissue wall of $O K C$ and $U A$ were viewed under polarization microscope and images were captured using three chip CCD cameras (Proview, media cybernetics, USA) with a $100 \times$ objective (oil immersion). Fiber thickness was determined on the images captured, using proplus image analysis software after proper calibration. All the measurements were in micrometers.

Polarization colors were determined separately for thin fibers (0.8 $\mu \mathrm{m}$ or less) and for thick fibers (1.6-2.4 $\mu \mathrm{m})$. In each section, five separate fields, in which there were at least 50 fibers of each size range were examined and the data from image analysis software was exported to M icrosoft excel master chartfor further interpretations and statistical analysis. 
Collagen fibers show yellowish-red to greenish-yellow birefringence under polarized microscope with picrosirius staining depending on thickness and packing. Usually, polarization colors of thin normal collagen fibers are green to greenish-yellow, whereas thick fibers range from yellowish to red birefringence. ${ }^{4}$

\section{RESULTS}

$68 \%$ of polarization colors of thin fibers of OKC were greenishyell ow. $47 \%$ of the thick fibers of OKC showed greenish-yellow color (Figs 1 and 3). Rest of the fibers showed yellowish-red color; $74 \%$ of polarization colors of thin fibers of UA were greenish-yellow and $31 \%$ of the thick fibers of UA showed greenish-yellow color (Figs 2 and 4). R est of the fibers showed yellowish-red color.

\section{Statistical Analysis}

Results are presented as frequency and percentage proportions. Z-test for proportions was used to compare the proportions

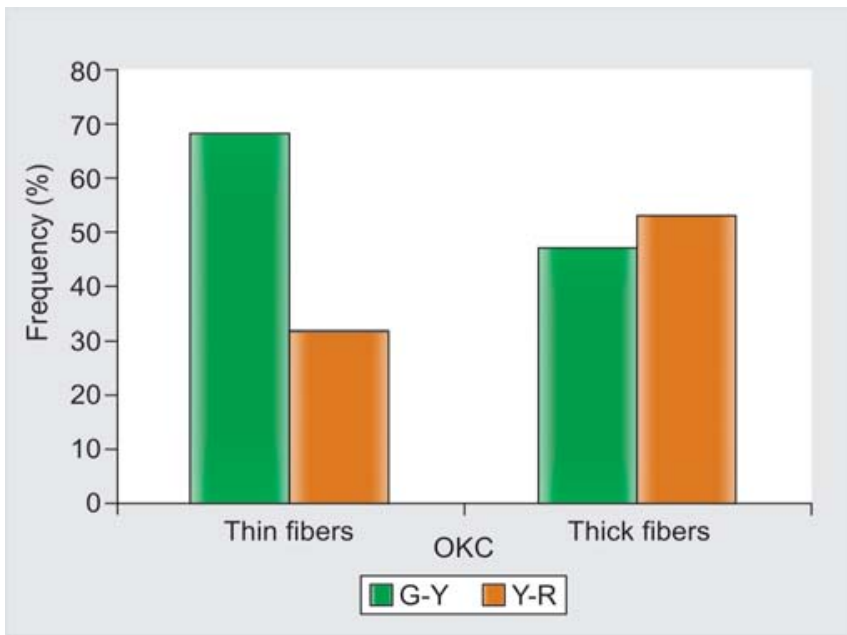

Fig. 1: Polarization colors of thin and thick collagen fibers in OKC

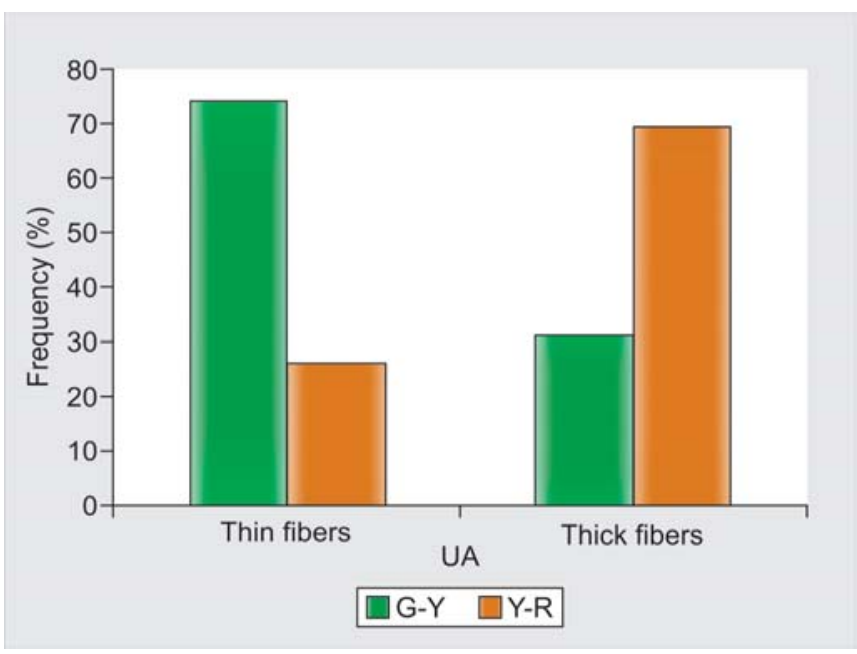

Fig. 2: Polarization colors of thin and thick collagen fibers in UA between the OKC and UA. A p-value of 0.05 or less was considered as statistically significant.

\section{DISCUSSION}

$\mathrm{UA}$ and $\mathrm{OKC}$ are clinically indistinguishable lesions. They both present as ordinary cysts in the dentate areas. ${ }^{5}$ The two entities are often difficult to distinguish, even though they have distinct histopathologic features. Variations in their prognosis and treatment modalities make it necessary to distinguish between them.

$\mathrm{OKC}$ is the most aggressive odontogenic cyst with high recurrence rate and a tendency to invade adjacent tissues, whereas $U A$, a variant of solid or multicystic ameloblastoma is less aggressive and has better prognosis than OKC as observed from previous, mainly experience-based, studies which estimated a recurrence rate of $25 \%{ }^{6}$ for $U A$ as opposed to 25 to $60 \%{ }^{7}$ for OKC.

In this study, we have tried to reassess the aggressive nature of the individual lesions based on histopathology by studying the polarization color of the collagen fibers in the cyst wall.

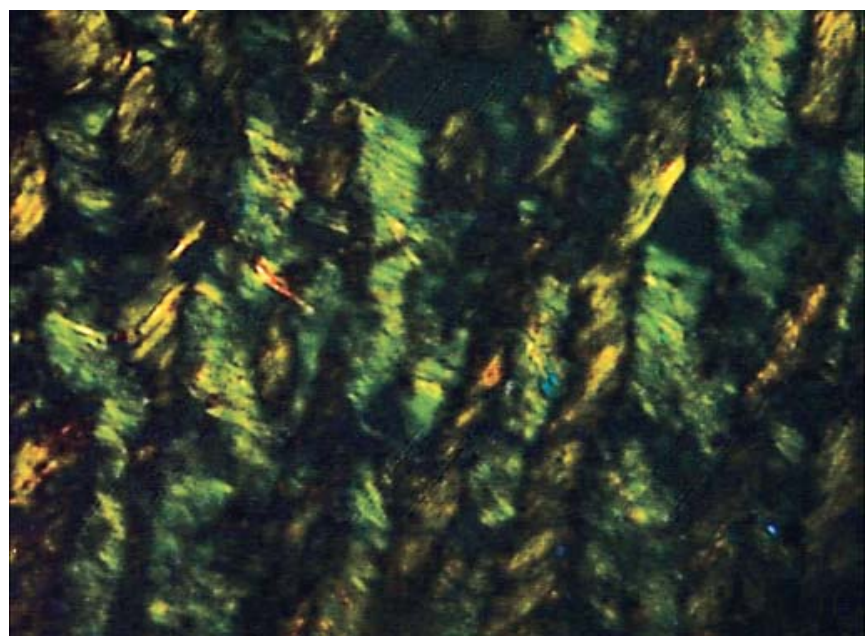

Fig. 3: Picrosirius red stained section of odontogenic keratocyst showing collagen fibers predominantly of greenish-yellow birefringence colors

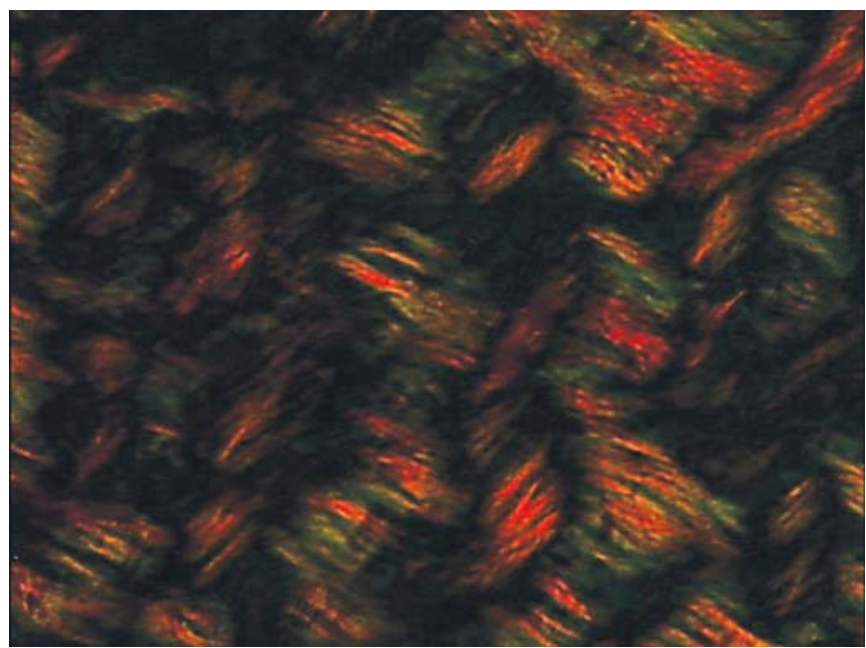

Fig. 4: Picrosirius red stained section of unicystic ameloblastoma showing collagen fibers of greenish-yellow and yellowish-red biref ringence colors 
Comparative Evaluation of Collagen Fibers in O dontogenic Keratocyst and Unicystic Ameloblastoma

It has been noted that biologic behavior of cysts and tumors is dependent not only on the epithelium present but al so on the stroma supporting the epithelium, and since collagen forms the major component of the stroma, the study of collagen fibers could be of diagnostic significance. ${ }^{8}$ Collagen has natural birefringence, which is attributed to the arrangement of its fibers. This property is enhanced by picrosirius red dye. Picrosirius red staining followed by polarizing microscopy can selectively demonstrate collagen. ${ }^{3}$

\section{Picrosirius Red Staining}

It is a strong anionic dye which stains collagen via its sulphonic acid group. Sirius red is an elongated dye. It attaches to the collagen fiber in such a way that their long axes are parallel to the long axis of each collagen molecule. This results in enlarged birefringence of the collagen.

The enhancement of birefringence promoted by the picrosirius polarization method is therefore specific for collagenous structures composed of aggregates of oriented molecules. $^{3}$

\section{Result with PSR Staining}

Tightly packed, better aligned collagen molecules give bright red-yellow strong birefringence.

Fibers composed of loosely packed thin fibrils give yell ow green weak birefringence.

In this study, polarization colors of the thin fibers $(0.8 \mu \mathrm{m}$ or less) were similar in both $U A$ and $O K C$. The polarization colors of the thick (1.6-2.4 $\mu \mathrm{m})$ collagen fibers in OKC were mostly green or greenish-yellow and those of UA were yellowish-red.

Previous studies have shown that polarization colors of picrosirius red-stained collagens are determined not only by fiber thickness but also by the packing of their constituent microfibrils. Tightly packed well-aligned fibers gives reddish-yellow color whereas loosely arranged thin fibers give greenish-yellow color. In our study, the greenish-yellow color of thick fibers in cases of OKC suggests that the collagen found in these lesions is loosely packed and might be composed of procollagens, intermediates, or other pathologic (not tightly packed) collagens. ${ }^{9}$

The loosely packed, denatured collagens are more susceptible to degradation by collagenase as well as other proteinases unlike normal collagen which has stable helical structure and is resistant to degradation. ${ }^{10}$ This feature would make OKC expand at a more rapid rate and may contribute to its aggressive nature.

A predominance of green to greenish-yellow color of thin and thick fibers has been found in several studies of different pathologic conditions using picrosirius red polarization method. The presence of green to greenish-yellow color of thick fibers was found in odontogenic tumors ${ }^{11}$ in humans and in an amel oblastic fibroma ${ }^{12}$ in a cat.
In the present study, in contrast to $O K C$, the thick fibers in the stroma of UA showed more of yellowish-red fibers. They correspond to normal tightly packed mature fibers. These findings were similar to the study conducted on odontogenic cysts, which included dentigerous cyst and radicular cyst, which also showed thick collagen fibers with reddish-yellow birefringence.

\section{CONCLUSION}

The present study was an attempt to assess the aggressive behavior of OKC and $U A$ by evaluating the nature of collagen fibers in the respective lesions. Presence of significantly high percentage of abnormally packed collagen fibers in OKC suggests that OKC is a more aggressive lesion in comparison with UA. Thus, the nature of collagen fibers as studied by the picrosirius red polarization method may be useful as a diagnostic tool to differentiate between the two lesions.

\section{REFERENCES}

1. Shear M. The aggressive nature of the odontogenic keratocyst: Is it a benign cystic neoplasm? (Part I). Clinical and early experimental evidence of aggressive behavior. Oral Oncology 2002;38:219-26.

2. Rajendran R. Cysts and tumors of odontogenic origin. In: Rajendran R, Shivapathasundaram (Eds). Shafer's Textbook of Oral Pathology (5th ed). New Delhi: Elsevier 2006;388.

3. J unqueira LCU, Bignolas G, B rentani RR. Picrosirius staining plus polarization microscopy, a specific method for collagen detection in tissue sections. Histochemical Journal 1979;11: 447-55.

4. Rabau MY Dayan. Polarization microscopy of picrosirius red stained sections: A useful method for qualitative evaluation of intestinal wall collagen. Histol Histopath 1994;9:525-28.

5. Chapelle KA, Stoelinga PJ W, V oorsmith RA. Rational approach to diagnosis and treatment of amel oblastomas and odontogenic keratocysts. B ritish J ournal of Oral and M axillofacial Surgery 2004;42:381-90.

6. Philipsen HP, Reichart PA. Unicystic ameloblastoma: A review of 193 cases from the literature. Oral oncology 1998;34(5): 317-25.

7. James RH. A review of odontogenic keratocysts and behavior of recurrences. Oral Surg Oral Med Oral Pathol Oral Radiol Endod 2006;101:5-9.

8. M arilena V, Izhar S, A mos B, Dayan D. M yofibroblasts in stroma of odontogenic cysts and tumors can contribute to variations in the biological behavior of lesions. Oral Oncol 2005;41(10): 1028-33.

9. Collagen fibers in the wall of odontogenic keratocysts: A study with picrosirius red and polarizing microscopy. J Oral Pathol M ed 1999:28:410-12.

10. Hand AR, Tencate A R. Cytoskel eton, junction and fibroblasts. In: Tencate AR (Ed). Oral Histology (5th ed). Singapore: Harcourt A sia Pvt Ltd 1999;61-78.

11. Hirshberg A, Buchner A, Dayan D. The central odontogenic fibroma and the hyperplastic dental follicle: Study with picrosirius red and polarizing microscopy. J Oral Pathol Med 1996:25:125-27.

12. Nyska A, D ayan D. A meloblastic fibroma in a young cat. J Oral Pathol M ed 1995;24:233-36. 